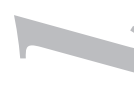

(n)
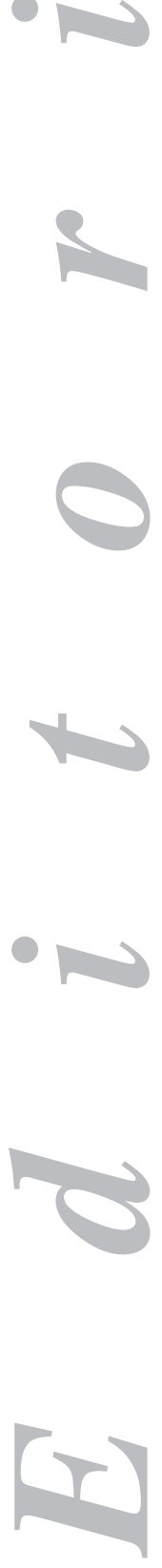

\section{Adult celiac disease - a common, significant health problem worldwide}

Celiac disease (CD) is an autoimmune systemic condition related to permanent gluten intolerance that develops in genetically predisposed individuals any time during their lifetime.

Mean prevalence worldwide is around $1 \%$ in most populations with no geographical, ethnical or racial differences, and the number of cases has notably increased during the last few decades. A greater understanding of the disease both in the adult and in children has contributed to this, as well as a higher availability of diagnostic methods, particularly serology.

While CD has been traditionally considered a childhood-onset disorders predominantly, it is now conclusively demonstrated that most patients are diagnosed when adults. Thus, recent studies, including one carried out in Finland that established the prevalence of $\mathrm{CD}$ in adults aged 50-70 years, have shown $\mathrm{CD}$ rates of $2.1 \%$, far higher than previously described (1).

Thus, CD recognition and an active search for cases should be encouraged at all ages, particularly in subjects of advanced age.

CD occurs worldwide but prevalence vary among countries. In the Asia-Pacific region prevalence ranges from 1:50 to 1:500 for serologic studies in adults, including countries as different as Australia, Iran, Israel, New Zealand, Syria and Turkey. In contrast, CD seems extremely rare in Japan, and was deemed to be rare in China until recently (2).

In India, as in most of Asia, prevalence is higher in northern states as compared to southern ones. Three factors may account for these geographical differences, including: a) a higher availability of wheat flour in some regions or countries because of historical or geographical reasons; b) a different distribution of known genetic susceptibility markers among the general population, mainly related to HLA-II, primarily subtype DQ2, which shows geographical, ethnical, and racial differences in worldwide distribution; c) a higher intake of gluten-containing foods because of changes in eating styles - regions traditionally with a rice-based diet have increasingly incorporated western-type food including a higher amount of products manufactured with wheat flour (bread and pasta).

All this is clearly reflected in the paper by $\mathrm{Wu} \mathrm{J}$ et al. (3) regarding a study performed in a Northern Chinese province where a serological screening of 78 individ- 
uals within two risk groups, namely type-I diabetics and patients with diarrhea-predominant irritable bowel syndrome, found a prevalence of $2.5 \%$, similar to that found in other studies performed in geographical areas as wide apart as Egypt, regarding a study in children (4). The explanations above fully warrant these findings.

$\mathrm{CD}$ has a wide spectrum of clinical manifestations, both intestinal and extraintestinal, given its autoimmune, systemic nature.

A recent study by Dr. Vivas et al. at Hospital de León, which analyzed differences in celiac disease expression between children and adults, confirmed that classic forms with predominant digestive symptoms and malabsorption develop in children within the first two years of life. From age 3 on atypical forms develop that are predominant in the adult. Female gender involvement predominates in both age groups with an approximate ratio of 2:1. Villous atrophy and high anti-tissue transglutaminase (tGT) antibody titers $(>100 \mathrm{U} / \mathrm{ml})$, clearly predominate in children and occur only exceptionally in adults (5).

In this issue of the Spanish Journal of Gastroenterology Dr. Fernández et al., at Hospital Povisa, Vigo, report on a long-term series of patients with adult CD. The authors confirm that the range of clinical manifestations is wide. In patients with refractory iron deficiency anemia or fluctuating high transaminase levels of unknown origin a differential screening of celiac disease is mandatory. They suggest that both atypical manifestations and a low suspicion index may delay the diagnosis for several years, even decades. They conclude by noting a marked increase in adult CD diagnoses during the last few years (6).

Adult CD occurs commonly associated with a number of disorders, which greatly enhances diagnosis by facilitating its recognition and prompt identification. The risk of having CD in first-grade relatives is estimated around 10-20\%, and may exhibit various clinical patterns, with this rate being somewhat lower in second-degree relatives. Therefore, given its clear genetic basis a family study is mandatory whenever a celiac patient is diagnosed $(7,8)$.

The disease is commonly associated with other endocrine conditions, and its frequency is increased in patients with type-I diabetes mellitus. Its diagnosis and a gluten-free diet (GFD), particularly in younger adults, improves diabetes control, decreases insulin needs, and increases bone mineralization (9). Accompanying thyroid disorders are also very common (both hyper- and hypothyroidism, with a higher prevalence of the latter), and normofunctioning autoimmune thyroiditis with positive anti-thyroid antibodies may also occur concomitantly. Of late tTG antibodies (tTGA) have been shown to deposit both in thyroid follicles and the extracellular matrix, and thus may play a pathogenic role in its development and maintenance (10).

Several studies performed both in Europe and the USA have suggested that iron deficiency anemia may be the first manifestation of CD in the absence of diarrhea, and is particularly suggestive in patients with chronic iron deficiency anemia that is refractory to therapy with oral iron (11). Chronic iron deficiency is very common in the general population. In young women it is often interpreted as a result of increased menstrual blood loss from abundant or frequent menses. 
Replacement therapy with oral iron usually results in partial or at least transient recovery depending on iron deficiency extent. However, in patients with advanced age responses are poorer or nonexistent, and thus the presence of anemia in postmenopausal women fairly commonly leads to a diagnosis of $\mathrm{CD}$ once the remaining causes of colonic or upper gastroduodenal bleeding have been ruled out (12).

Gynecological and fertility disturbances in women are common and significant in patients with $\mathrm{CD}$, and should be systematically investigated in females with associated recurrent digestive complaints, since correction is effective and sustained with a maintenance GFD following diagnosis. Similarly, males may also present with celiac-related sexual issues, including infertility and libido problems whose improvement and correction require an adequately established diagnosis (13).

Among neurological diseases cerebellar ataxia is commonly associated with $\mathrm{CD}$, particularly when onset occurs at advanced age. Various pathogenic immune mechanisms have been described, as well as a number of gluten-related antibodies that may cross the blood-brain barrier and deposit in Purkinje cells, where they give rise to a severe inflammatory response with progressive neuronal destruction (14). Response to a GFD is usually more effective when it is initiated within six months after onset; results are poorer thereafter. A tTG isoenzyme, namely subtype 6 , has been recently shown to occur with greater frequency in patients with CD-related ataxia, and its positivity would therefore help establish the causal diagnosis of the associated neurological disturbance (15).

The prevalence of hypertransaminasemia (HT) is increased in both children and adults with CD. Thus a study by a group of pediatricians in Barcelona, which included a total of 114 children with CD, $60 \%$ with classical forms and $40 \%$ with atypical manifestations, the authors found moderate HT at diagnosis in $32 \%$ of patients, and this was the only sign for 5 subjects (4.3\%). Patients with HT were usually younger and had classic forms predominantly when compared to those with normal levels. Aminotransferase values returned to normal with GFD in all cases after a minimal follow-up of 1 year (16). A significant percentage of nonalcoholic fatty liver disease (NAFLD) cases with no associated metabolic risks may in fact derive from undiagnosed $\mathrm{CD}$. Thus, Bardella et al. found in a series of 60 adult patients with HT that six of them had positive tTG (10\%). After six months on GFD liver enzymes returned to normal in all subjects (17).

$\mathrm{CD}$ is commonly associated with various skin and mucosal disorders that present as bouts or flare-ups, and that usually are highly pruriginous. Most common and typical among them is dermatitis herpetiformis (DH), which is characterized by initial papulovesicular lesions that later become crusty and may affect several body areas; their distribution is usually symmetrical and involves both knees, buttocks, and wrists, but lesions are also highly typical in the face, hands and scalp. A skin biopsy collected from the vicinity of lesions will show highly characteristic linear IgA deposits at the epidermal basal layer. The rate of $\mathrm{DH}$ is high as it occurs in up to $25 \%$ of celiac patients, and its presence unequivocally signals the presence of $\mathrm{CD}$. Gastrointestinal complaints may be mild or absent given that duodenal lesions are usually mild; their presence greatly facilitates a di- 
agnosis of gluten intolerance, and these symptoms are considered the condition's visiting card. Furthermore, $100 \%$ of patients with DH should be considered celiac individuals (18).

Patients with CD have a 70-fold higher risk of developing microscopic colitis versus the general population (19). Similarly, its prevalence in patients meeting irritable bowel syndrome criteria is more than 4 times higher than in controls, as was confirmed by a recently reported meta-analysis on this topic (20).

We may conclude by pointing out that $\mathrm{CD}$ is a significant health issue worldwide, and one that is clearly underestimated and underdiagnosed at that (only $10 \%$ of potential cases have been identified). Improved clinical status requires a deeper understanding of this multifaceted condition, and a multidisciplinary approach that increasingly relies on close collaboration among all practitioners involved (primary care physicians, gastroenterologists, hematologists, gynecologists, dermatologists, neurologists, rheumatologists, surgeons and internists, among others).

L. Rodrigo-Sáez and I. Pérez-Martínez

Service of Digestive Diseases. Hospital Universitario Central de Asturias (HUCA). Oviedo, Asturias. Spain

\section{REFERENCES}

1. Vilppula A, Kaukinen K, Luostarinen L, Krekelä I, Patrikainen H, Valve R, et al. Increasing prevalence and high incidente of celiac disease in elderly people : a population-based study. BMC Gastroenterol 2009; 9: 49.

2. Cummins AG, Roberts-Thomson IC. Prevalence of celiac disease in the Asia-Pacific region. J Gastroenterol Hepatol 2009; 24: 1347-51.

3. Wu J, Xia MB, von Blomberg MB, Zhao C, Yang XW, Crusius JBA, et al. Coeliac disease in China, a field waiting for exploration. Rev Esp Enferm Dig 2010: 102(8): 472-7

4. Abu-Zekry M, Kryszak D, Diab M, Catassi C, Fasano A, et al. Prevalence of celiac disease in Egyptian chidren diputes the east-west agriculture-dependent spreads of the disease. J Pediatr Gastroenterol Nutr 2008; 47 : $136-40$.

5. Vivas S, Ruiz de Morales JM, Fernández M, Hernando M, Herrero B, Casqueiro J, et al. Age-related clinical serological and histopathological features of celiac disease. Am J Gastroenterol 2008; 103: 2360-5; quiz 2366.

6. Fernández A, González L, De la Fuente J. Celiac disease: clinical features in adult populations. Rev Esp Enferm Dig 2010; 102(8): 466-71.

7. Rodrigo L, Riestra S, Fuentes D, González S, López Vázquez A, López Larrea C. Diversas formas de presentación clínica de la enfermedad celíaca dentro de la misma familia. Rev Esp Enferm Dig 2004; 96: 612-9.

8. Neuhausen SL, Steele L, Ryan S, Mousari M, Pinto M, Ossan KE, et al. Co-occurrence of celiac disease and other autoinmune diseases in celiacs and their first-degree relatives. J Autoimmun 2008; 31: 160-5.

9. Diniz-Santos DR, Brandão F, Adan L, Moreira A, Vicente EJ, Silva LR. Bone mineralization in young patients with type 1 diabetes mellitus and screening-identified evidence of celiac disease. Dig Dis Sci 2008; 53 : $1240-5$.

10. Naiyer AJ, Shah J, Hernandez L, Kim SY, Ciaccio EJ, Cheng J, et al. Tissue transglutaminase antibodies in individuals with celiac disease bind to thyroid follicles and extracellular matrix and may contribute to thyroid dysfunction. Thyroid 2008; 18: 1171-8.

11. Corazza GR, Valentini RA, Andreani ML, D'Anchino M, Leva MT, Ginaldi L, et al. Subclinical coeliac disease is a frequent cause of iron-deficiency anaemia. Scand J Gastroenterol 1995; 30: 153-6.

12. Ransford RA, Hayes M, Palmer M, Hall MJ. A controlled, prospective screening study of coeliac disease presenting as iron deficiency anemia. J Clin Gastroenterol 2002; 35: 228-33.

13. Sanders DS. Coeliac disease and subfertility: association is often neglected. BMJ 2003; 327: 1226-7.

14. González S, Rodrigo L, Salas-Puig J, Astudillo A, Fuentes D, López-Vázquez A, et al. Ataxia mioclónica progresiva asociada con anticuerpos frente a las células de Purkinje en un paciente celíaco. Rev Esp Enferm Dig 2005; 97: 918-21. 
15. Hadjivassiliou M, Aeschlimann P, Strigun A, Sanders DS, Woodroofe N, Aeschlimann D. Autoantibodies in gluten ataxia recognize a novel neuronal transglutaminase. Ann Neurol 2008; 64: 332-43.

16. Farré C, Esteve M, Curcoy A, Cabre E, Arranz E, Amat LL, et al. Hypertransaminasemia in pediatric celiac disease patients and its prevalence as a diagnostic clue. Am J Gastroenterol 2002; 97: 3176-81.

17. Bardella MT, Valenti L, Pagliari C, Peracchi M, Fare M, Fracanzani AL, et al. Searching for coeliac disease in patients with non-alcoholic fatty liver disease. Dig Liver Dis 2004; 36: 333-6.

18. Collin P, Reunala T. Recognition and management of the cutaneous manifestations of celiac disease: a guide for dermatologists. Am J Clin Dermatol 2003; 4: 13-20.

19. Green PH, Yang J, Cheng J, Lee AR, Harper JW, Bhagat G. An association between microscopic colitis and celiac disease. Clin Gastroenterol Hepatol 2009; 7: 1210-6.

20. Ford AC, Chey WD, Talley NJ, Malhotra A, Spiegel BM, Moayyedi P. Yield of diagnostic tests for celiac disease in individuals with symptoms suggestive of irritable bowel syndrome: systematic review and meta-analysis. Arch Intern Med 2009; 169: 651-8. 